\title{
Simultaneous Inpainting and Motion Estimation of Highly Degraded Video-Sequences
}

\author{
Jean Pierre Cocquerez ${ }^{* 1}$, Laurent Chanas ${ }^{2}$, Jacques Blanc-Talon ${ }^{3}$ \\ 1 Universite de Technologie de Compiegne(UTC), 60205 Compiegne, France. \\ (cocquerez@hds.utc.fr), \\ 2 Vision IQ, 2bis rue National 92100 Boulogne, France, (laurent.chanas@free.fr) \\ 3 GIP department, CTA, 16 av Prieur de la Cte d'Or 94000 Arcueil, (blanc@etca.fr)
}

\begin{abstract}
This article deals with new restoration algorithms of strongly degraded videos. Degradations appears either as missing data in every image or as a large magnitude impulse noise. The proposed algorithm is based upon a partial differential equation formalism coming from recent works on image inpainting and video sequence restoration. It use a $3 \mathrm{D}$ representation of the video sequences and can process moving background sequence. Simultaneous inpainting and motion estimation are carried out. High quality results are presented in the context of the restoration of degraded films.
\end{abstract}

\section{Introduction}

This article deals with restoration of highly degraded video sequences for which there is a lot of missing data. Applied on this kind of highly degraded images, the recent restoration algorithms do not give satisfactory results. On the one hand, the spatial inpainting methods [1] cannot be used directly because there is not enough reliable information in one image. More precisely, the missing regions are generally larger than the observed objects. On the other hand, the temporal filtering methods (see section 1.1) are based on a temporal evolution model which can be applied if a pixel is valid at least in one image. Therefore, we introduce a new algorithm that restores highly degraded image sequences with large spatial and temporal missing data areas and computes simultaneously the optical flow.

\subsection{Background}

Processing of image sequences often use the temporal continuity of images to improve restoration. The following approaches [2] can be distinguished:

Purely temporal filtering: a monodimensional filtering is applied independently to each pixel along the temporal axis[3].

Temporal filtering with motion compensation: monodimensional filtering is applied to the pixels which belong to the same object in every image. To improve results, a motion estimation is calculated to perform filtering along the object trajectory, [4][5]. 
Spatiotemporal filtering with motion compensation : Spatial and temporal filtering allows to take into account the spatial discontinuities. Several approaches have been carried out : spatial segmentation of objects [6], threedimensional Wiener filter [7], Kalman filter [8], wavelet transform [9]. S Geman, D.E. Mc Clure and D. Geman [10] use the Bayesian formalism to restore small deteriorations in films.

Spatio temporal filtering with motion estimation: Filtering quality depend on the quality of the motion estimation, and vice versa. Consequently, a good approach might be to perform simultaneously denoising and motion estimation. Thus, Kokaram uses an auto-regressive 3D model [7] and proposes a method which simultaneously detects artefacts, calculates motion parameters and restores images, whereas Fannuses a bayesian formulation [11]. Kornprobst [12] uses a variational approach for extracting moving objects and restoring the background simultaneously.

\subsection{Choice of an approach}

Our application is dedicated to strongly degraded films. Degraded pixels are saturated (see section 3) and without information. Sequences contain a lot of degraded zones whose spatial and temporal dimensions can be important. Restoring the missing data from a lonesome image is too difficult. Notes that the position of the degradeted area are supposed to be known.

The previous algorithms might be inefficient if they are straightforwardly applied to highly degraded image sequences. However, their principles can be taken into account to solve our problem. Two remarks explain our approach: a pixel is not degraded in the whole image sequence, the value of a degraded pixel might be computed from its neighborhood. So, we decided to carry out a tridimensional diffusion mechanism on the reliable pixels. Applying a variational approaches allows to take efficiently into account the diffusion mechanisms.

\section{Inpainting of video sequences}

In our application,the temporal and spatial motion models are supposed identical. The image sequence is considered as a volume $I$. We denote $u_{0}$ : the degraded image sequence and $u$ : the restored image sequence.

From a mathematical point of view, $u$ and $u_{0}$ are piecewise continuous functions of $\mathbb{R}^{3} \rightarrow \mathbb{R}$ defined in the space of bounded variations $B V$. Taking into account that the gray level is constant along the path given by the optical flow, we propose to compute the optical flow in order to carry out a motion compensation. Inspired by previous works of Geman [10], Kokaram [7] and Kornprobst [13], we present a new expression of the energy (see equation 1) whose minimization allows four processings to be simultaneously performed : a spatial diffusion, a denoising, a temporal filtering with motion compensation and motion estimation. 


$$
\begin{aligned}
& E_{f o}(u, \sigma)=\underbrace{\int_{I} c\left(u-u_{0}\right)^{2} d X+\lambda_{1} \int_{I} \phi_{1}(|\nabla u|) d X}_{1}+\underbrace{\lambda_{2} \int_{I} \phi_{2}\left(\sigma \cdot \nabla u+\frac{\partial u}{\partial t}\right) d X}_{2} \\
& +\underbrace{\lambda_{3} \int_{I} \phi_{3}\left(\left|\nabla \sigma_{x}\right|\right) d X+\lambda_{3} \int_{I} \phi_{3}\left(\left|\nabla \sigma_{y}\right|\right) d X}_{3}
\end{aligned}
$$

The part 1 of the equation (1) is a restoration term based on a two-dimensional spatial diffusion applied on each frame of the sequence. This part is composed of two term: an attach term to the data $(c=1$ if the voxel $(x, y, t)$ is valid) and an anisotropic diffusion term that inpaints the images within the missing data areas. The part 2 has two meanings :-from the image processing point of view, it's a regularization term depending on the optical flow whose aim is to perform temporal filtering with motion compensation, -associated with the part 3 , it allows a robust computation of the optical flow. Indeed, we recognize a part of the optical flow equation:

$$
\sigma . \nabla u+\frac{\partial u}{\partial t}
$$

where $\sigma=\left(\sigma_{x}, \sigma_{y}\right)^{t}$ denotes the velocity and $u(x, y, t)$ the current voxel.

The third part is a regularization of the motion vector. Functions $\phi_{i}()$ are choose identical to $\phi(z)=z$ in order to carry out an anisotropic diffusion.

\subsection{Convergence}

The equation (1) can be written according to the following general form:

$$
\begin{aligned}
E_{g}(u, \sigma) & =\int_{I}\left(u-u_{0}\right)^{2} c d X+\lambda_{1} \int_{I} \phi_{1}(|\nabla u|) d X \\
& +\lambda_{2} \int_{I} \phi_{2}\left(\sigma . \nabla u+\frac{\partial u}{\partial t}\right) d X+\lambda_{3} M(\sigma)
\end{aligned}
$$

where $M(\sigma)$ denotes a velocity regularization. For a given $\sigma_{0}$, it can be proven that $E_{g}\left(u, \sigma=\sigma_{0}\right)$ admits a unique minimum $u_{m}$. Indeed, this energy is the sum of continuous and convex terms. For a given $u_{0}, E_{g}\left(u=u_{0}, \sigma\right)$ admits also a unique minimum. The proof is given in Kornprobst's dissertation [14] for the regularized optical flow, or in Black's dissertation [15] for affine motion. So, we can assert that each of the minimizations has a unique solution (except when $\phi_{i}(x)=x$, for which that minimum may not be unique)[16]. Consequently, the equation (1) has a minimum $(u, \sigma)$, but as this energy is not convex with respect to $(u, \sigma)$, the solution is not unique. 


\subsection{Solving}

The minimization of the energy (1) is performed according three steps: first, a two-dimensional rough restoration is performed to approach the solution and to avoid local minima, then, a minimization with respect to $\sigma$ and a minimization with respect to $u$ are carried out.

The rough restoration is performed according to the spatial filtering proposed by Chan and Shen [17], which consists in setting $\lambda_{2}=\lambda_{3}=0$ in the equation (3)

Minimization with respect to the optical flow. This minimization takes place for a fixed $u$. Applying the Euler-Lagrange's theorem on equation (1) gives:

$$
\begin{gathered}
\forall i \in[x, y] \\
\lambda_{3} . \operatorname{div}\left(\frac{\phi_{3}^{\prime}\left(\left|\nabla \sigma_{i}\right|\right)}{\left|\nabla \sigma_{i}\right|} \nabla \sigma_{i}\right)=\frac{\phi_{2}^{\prime}\left(\sigma . \nabla u+\frac{\partial u}{\partial t}\right)}{\sigma . \nabla u+\frac{\partial u}{\partial t}}\left(\sigma . \nabla u+\frac{\partial u}{\partial t}\right) u_{i} .
\end{gathered}
$$

The equation (4) is solved by using the auxiliary following variables :

$d \zeta_{i}=\frac{\phi_{3}^{\prime}\left(\left|\nabla \sigma_{i}\right|\right)}{\left|\nabla \sigma_{i}\right|}$ and $d \vartheta=\frac{\phi_{2}^{\prime}\left(\sigma \cdot \nabla u+\frac{\partial u}{\partial t}\right)}{\sigma \cdot \nabla u+\frac{\partial u}{\partial t}}$

Then, the energy is minimized alternately with respect to $\left(d \zeta_{x}, d \zeta_{y}, d \vartheta\right)$ and $\sigma$. Because the optical flow equation (2) can be only used in presence of subpixel displacement, the computation of the optical flow is performed with a multiscale scheme. Details about the discretization of this kind of equation can be found in [14].

Minimization with respect to the variable $\boldsymbol{u}$ The energy (3) contains two non-quadratic terms with respect to $\mathrm{u}$. Minimization is performed using semi-quadratic method proposed by Charbonnier [18],[19]. Due to the GemanReynolds's theorem, energy can be expressed under a quadratic form by incorporating two functions $\Psi_{1}$ and $\Psi_{2}$ so that its minimization becomes simpler to solve.

The energy:

$$
\begin{aligned}
& \int_{I}\left(u-u_{0}\right)^{2} c d X+\lambda_{1} \int_{I} \phi_{1}(\|\nabla u\|) d X \\
& +\lambda_{2} \int_{I} \phi_{2}\left(\sigma . \nabla u+\frac{\partial u}{\partial t}\right) d X
\end{aligned}
$$

becomes an energy function of $u, b_{1}$ and $b_{2}$

$$
\begin{aligned}
& \int_{I}\left(u-u_{0}\right)^{2} c d X+\lambda_{1} \int_{I}\left(\Psi_{1}\left(b_{1}\right)+b_{1}|\nabla u|^{2}\right) d X \\
& +\lambda_{2} \int_{I}\left(\Psi_{2}\left(b_{2}\right)+b_{2}\left(\sigma \cdot \nabla u+\frac{\partial u}{\partial t}\right)^{2}\right) d X
\end{aligned}
$$


The values of $b_{1}$ and $b_{2}$ which minimize the energy (6) are also given by the Geman-Reynolds's theorem. The minimization consists in computing the following equations alternatively (in which we used the relation $\frac{d u}{d t}=\sigma . \nabla u+\frac{\partial u}{\partial t}$ ):

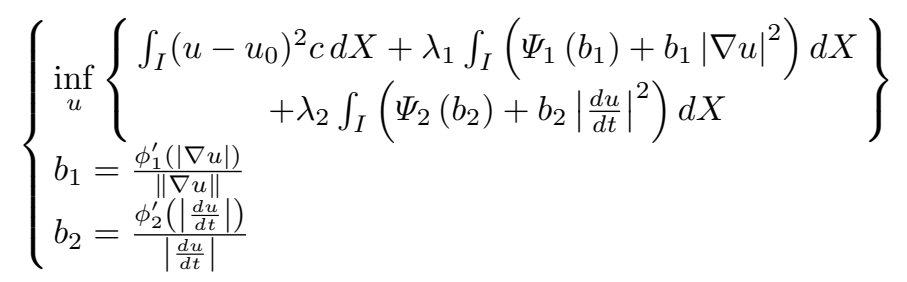

For a given $\sigma$,the minimization of the first part of the equation (7) according to Euler-Lagrange's theorem gives:

$$
c\left(u-u_{0}\right)-\lambda_{1} \cdot \operatorname{div}_{x y}\left(b_{1} \nabla u\right)-\lambda_{2} \cdot \operatorname{div}_{x y t}\left(b_{2}\left|\frac{d u}{d t}\right| \theta\right)=0
$$

where $\theta=\left(\sigma_{x}, \sigma_{y}, 1\right)^{t}$ denotes the velocity in the tridimensional space $(\mathrm{x}, \mathrm{y}, \mathrm{t})$.

Using motion compensation complicates the algorithms, because, besides the parameters $\lambda$ of the energy, the calculation of the motion estimation requires several other parameters. For instance, the computation of the regularized optical flow requires to choose a function $\phi($.$) , and the number of levels of the multiscale$ pyramid (which is set to 2 in our application).

With respect to the value of the three parameters $\left(\lambda_{1}, \lambda_{2}, \lambda_{3}\right)$, we chose to perform similar temporal and spatial regularizations $\left(\lambda_{2}\right)$. $\lambda_{1}$ is chosen according to either the thinness of detail having to be preserved, or according to the strength of the denoising. Typically, $\lambda_{1}$ is fixed within the range 1 and 100. $\lambda_{3}$ is fixed to $\lambda_{3}^{\prime}=\frac{\lambda_{3}}{\lambda_{2}}$.

\section{$3 \quad$ Experimental results}

We processed a video sequence given by the INA ${ }^{4}$. Defaults consist in a vertical strip and large white spots corresponding to film degradations. We present the first two frames of the degraded image sequence (figure 1) and the corresponding restored frames (figure 2). On the figure 3 we present the estimated motion vector around the human which appears, from the left, in the background of the scene. Computing time is about 30 seconds per frame (720x526pixels) for a pentium4 PC (1.5GhZ), This time is acceptable for an off line inpainting process. At present, our algorithm is not optimized, the sequence is split into blocks of 10 frames which are processed sequentially

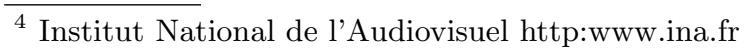




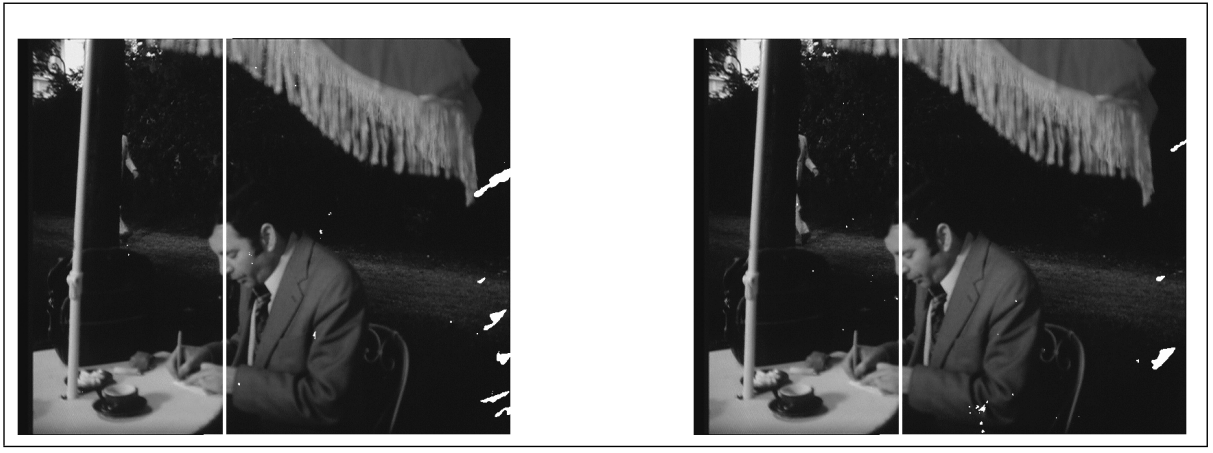

Fig. 1. degraded sequence

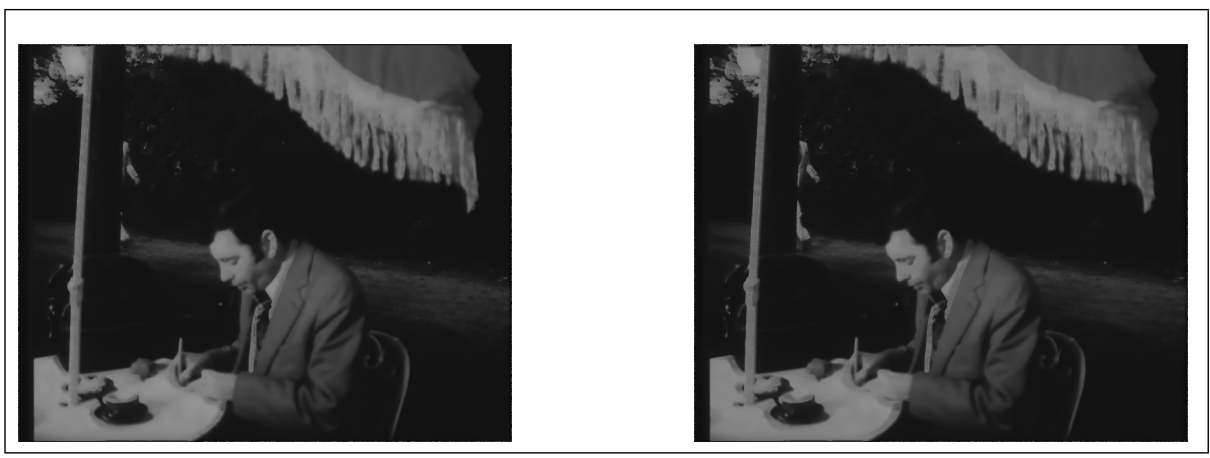

Fig. 2. restored sequence

\section{Conclusion}

In this paper, we have studied the general problem of the restoration of highly degraded image sequences. The choice of the variational approach is very positive. Indeed, on the one hand, this approach is unifying one, in the sense where links can be established with the other approaches and on the other hand the anisotropic diffusion which constitutes the base of the chosen approach, allows simultaneous reduction of noise and to reconstruction of the lost information. Then, to take into account the camera displacement, we proposed a new energy allowing to carry out simultaneously the optical flow calculation, the restoration, and the images reconstruction.Results are very promising

\section{References}

1. Masnou, S., Morel, J.M.: Level-lines based disocclusion. In: Proceedings of 5th IEEE Int'l Conf. on Image Process., Chicago (1998) 3:259-263 


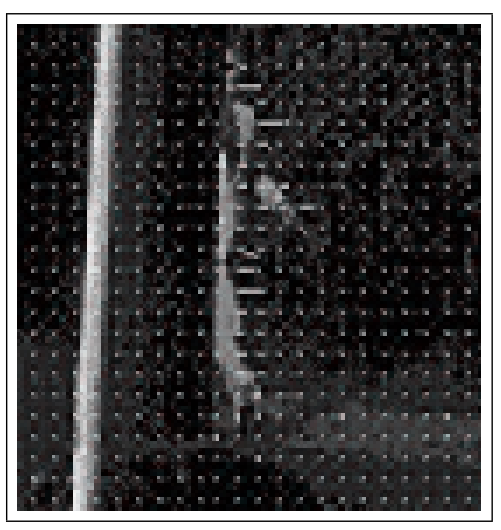

Fig. 3. optical flow(around the human which appears, from the left to the right, in the background of the scene)

2. Dekeyser, F., Bouthemy, P., Prez, P.: Spatio-temporal wiener filtering of images using a parametric motion model. In: Proceedings of the IEEE International Conference on Image Processing, ICIP'2000, Vancouver, British Columbia (2000)

3. Chanas, 1., Cocquerez, J.P., Blanc-Talon, J.: Numerical energy minimization for removing high luminous energy in dazzled images. Advanced Concepts for Intelligent Vision Systems (ACIVS'99), Baden-Baden, Germany (1999)

4. Kleihorst, R., De Haan, G., Lagendijk, R., Biemond, J.: of Signal Processing VI : Theories and Applications. In: Motion compensated noise filtering of image sequences. Elsevier science (1992) 1385-1388

5. Giaccone, P., G.A., J., Minelly, S., Curley, A.: Motion-compensated multi-channel noise reduction of colour film sequences. SPIE Journal of Electronic Imaging 8 (99) 246-254

6. Kalivas, D., Sawchuk, A.: Motion compensated enhancement of noisy image sequences. In: Proceedings of the IEEE International conference on acoustics, speech and signal processing, Albuquerque (1990) 2121-2124

7. Kokaram, A.: Motion Picture Restoration. Springer-Verlag London (1998)

8. Woods, J., Kim, J.: 12. In: Motion compensated spatio-temporal kalman filter. Kluwer Academic (1993) 349-373

9. VanRoosmalen, P.M.B., Westen, S.J.P., Lagendijk, R.L., Biemond, J.: Noise reduction for image sequences using an pyramid thresholding technique. In: Proceedings of the IEEE International Conference on Image Processing, Lausanne (1996) 375378

10. Geman, S., McClure, D., Geman, D.: A nonlinear filter for film restoration and other problems in image processing. Graphical models and image processing $\mathbf{5 4}$ (1992) 281-289

11. Fan, C., Namazi, N.: Simultaneous motion estimation and filtering of images sequences. IEEE Transactions on image processing 8 (1999) 1788-1795

12. Kornprobst, P., Deriche, R., Aubert, G.: Image sequence restoration : a pde based coupled method for image restoration and motion segmentation. In: Proceedings of the European Conference on Computer Vision, Freiburg, Allemagne (1998) 548562 
13. Aubert, G., Deriche, R., Kornprobst, P.: Computing optical flow via variational techniques. SIAM Journal on Applied Mathematics 60 (2000) 156-182

14. Kornprobst, P.: Contribution la restauration d'images et l'analyse de squences : Approches Variationnelles et Solutions de Viscosit. $\mathrm{PhD}$ thesis, Universit de Nice-Sophia Antipolis (1998)

15. Black, M.J.: Robust incremental optical flow. PhD thesis, Yale university (1992)

16. Chanas, L.: Methodes variationnelles pour la restauration de squences d'images fortement degradees. PhD thesis, Cergy University (2001)

17. Chan, T., Shen, J.: Non-texture inpainting by curvature-driven diffusions (ccd). Technical report, UCLA CAM Report (2000)

18. Charbonnier, P., Aubert, G., Blanc-Ferraud, M., Barlaud, M.: Two-deterministic half-quadratic regularization algorithms for computed imaging. In: Proc. IEEE int. Conf. on Image Proc. ICIP. Volume 1., Austin (Texas) (1994) 168-172

19. Charbonnier, P., Aubert, G., Blanc-Ferraud, M., Barlaud, M.: Deterministic edgepreserving regularization in computed imaging. IEEE Transactions on Image Processing 6 (1997) 298-311 